Electronic Optical Astronomy: Philosophy and Practice Author(s): Edwin W. Dennison

Source: Science, New Series, Vol. 174, No. 4006 (Oct. 15, 1971), pp. 240-244

Published by: American Association for the Advancement of Science

Stable URL: http://www.jstor.org/stable/1732153

Accessed: 14/11/2014 12:38

Your use of the JSTOR archive indicates your acceptance of the Terms \& Conditions of Use, available at

http://www.jstor.org/page/info/about/policies/terms.jsp

JSTOR is a not-for-profit service that helps scholars, researchers, and students discover, use, and build upon a wide range of content in a trusted digital archive. We use information technology and tools to increase productivity and facilitate new forms of scholarship. For more information about JSTOR, please contact support@jstor.org. 


\section{Electronic Optical Astronomy: Philosophy and Practice}

\author{
Observational techniques of optical astronomy are being \\ effectively changed by modern electronic instruments.
}

Edwin W. Dennison

\section{Introduction}

Throughout the history of observational optical astronomy the observer has always been in a state of anxious frustration because he almost always chooses to work on problems which require that his instruments operate at the limit of their capability. Under these conditions he is always vulnerable to systematic errors introduced by his observing instruments. Each new technique brings to the observer the new power that he needs to advance his understanding of the universe, but at the same time he must check and recheck so that he can be sure that a new instrument does not give him erroneous data. The introduction of large telescopes and the photographic plate required a period of testing before they became generally acceptable. The application of electronic instruments to observational astronomy, which began about the middle of the 20 th century, is no exception. This revolution caused by electronic instrumentation is still in progress, and will continue to alter astronomical observing technology as long as new devices and components are developed and perfected.

\section{Goals and Philosophy}

As with most areas of scientific endeavor, there is a cyclic relation between philosophy and practice, with each in turn leading to a new development in the other. At this time we are following the philosophy that new elec-

The author is head of the Astroelectronics Laboratory at the Hale Observatories and a research associate at the California Institute of Technology, pasadena 91109. because of equipment failure and the introduction of systematic errors that result from malfunctioning of the equipment.

The designer of electronic systems must understand the special relationship between an observer and his equipment. This understanding must include the conditions of fatigue and discomfort under which the observer operates. The designer must guard against the introduction of systematic errors resulting from observer bias. All controls for a complex electronic instrument must be designed to provide the observer with only those controls that he needs to operate his equipment during the night. Adjustment controls and controls that are set for each observing run must be located at a position that is not immediately accessible to the observer or night assistant. As far as possible, the controls must be self-explanatory because there are often long periods of time between each observing assignment. The controls must be simple and self-protecting to avoid the possibility that the observer will introduce errors. The future development of electronic instrumentation will lead to more systems that must be operated by the observer and night assistant. If each set of controls is not scrutinized to provide simple operation, the observer may soon be confronted with a large number of complex controls that make his observing tasks almost impossible.

To avoid systematic errors, the observer must never be a link in the data-collection chain; however, there must be adequate provision for him to monitor all of the vital instrument functions. The observer must always have complete control over his data system to ensure that the data are collected as he wishes and to eliminate the feeling that he is being manipulated by the dictates of the equipment.

It is important to recognize the conditions under which an observer can make optimum use of his telescope observing time. For routine observations of a series of objects there is no necessity for the observer to see more than partially reduced data. Under these circumstances the observer's most important function is to ascertain that the objects are correctly identified and that the data-gathering process is proceeding in a valid manner. When the observer is looking at new and previously unexplored objects, it is vital that he see data reduced as fully as possible so that he can determine the length of time and the appropriate instrumental 
conditions that he must use. Experienced observers are able to interpret fragmentary data with a high degree of effectiveness. If the observer detects some photometric or spectroscopic feature that appears to be interesting, he can repeat the observations immediately and thereby confirm or refute his initial impression. Thus the observer can spend more time substantiating interesting observations and less time making observations that will be discarded later. It is impossible for any machine or automatic device to have the flexibility and ingenuity of an experienced observer.

Having explored the philosophical basis for modern electronic instrumentation, I now describe some of the instruments that have been built at the Hale Observatories. These instruments have been built over a period of many years, and our philosophy of instrument design has evolved from our experience. I will emphasize those instruments now under development because they exemplify current thinking.

\section{Reduction Equipment}

Microphotometers are the basic instruments for measuring the densities of photographic plates to obtain quantitative photometric information about astronomical images. The classical microphotometer is a carefully designed mechanical and optical instrument in which two slits are used to minimize the effects of scattered light in the photographic emulsion. Usually these have been equipped with d-c amplifiers and strip-chart recorders. Many optical, mechanical, and electronic devices have been designed and built to transform the photographic-plate densities into exposure information. With the advent of small computers, it is now possible to perform the function transformation and data-reduction process more rapidly and more accurately. Control of the mechanical operation of the microphotometer can be done by the computer. Computer-controlled microphotometers have resulted in enormous savings in time and manpower required for data reduction.

Microphotometers with variable iris diaphragms projected on the photographic plate, with either manual or automatic operation of the iris movement, have been used to measure the diameter and thus the brightness of stellar images. The iris position and the mechanical motions can be read by encoders and recorded on punch cards or punch paper tape. Devices of this type speed the measurement process and minimize the chance for human error. Similarly, measuring engines for determining the positions of stellar images or spectral lines can be equipped with automatic data-recording devices.

\section{Aids That Do Not Gather Data}

Sophisticated electronic observing instruments and automatic data-collection systems can enhance observing effectiveness by large factors, but there are other devices that can increase telescope and observer operating efficiency. These devices are not part of the datacollection system but rather are aids to the observer. Automatic dome and windscreen controlling devices are one example. Computer-controlled telescope-setting systems can also aid significantly if the computer program includes corrections for atmospheric refraction, telescope flexure, the errors in apparent stellar positions produced by the orbital motion of the earth, and corrections for the precession of the star position. If all of these corrections are included in a computer operating system, the telescope can be set on any previously known object to an accuracy of better than 10 seconds of arc. Such systems enable an observer to go directly to any object without the time-consuming process of searching through the field of nearby stars to ascertain that he is accurately set on the object he wishes. Digital exposure meters can be of great assistance to observers using coudé spectrographs. These devices can permit the observer to obtain uniform exposures regardless of variations in image quality and atmospheric transparency.

Many modern observational problems are concerned with photometric and spectroscopic measurements of extremely faint objects that are obtained with instruments that dynamically subtract the sky background. Such objects can be too faint to be seen with the unaided eye. In the past, faint objects of this type have been located by an initial, long photographic exposure, generally at the same focal position of the telescope, and then by measuring the position of the desired object with respect to brighter stars in the neighboring stellar field. By means of precision screws on the telescope, the observer must set his instrument at the position of the invisible object with respect to the brighter stars. This can be a very time-consuming process. Preliminary experiments indicate that by the use of integrating television cameras coupled with intensifiers it is possible to display objects which are approximately ten times fainter than those which can be seen by the unaided eye. If the observer is able to see these faint objects displayed on a television screen, he can compare the fields directly with existing sky charts, such as the Palomar 48-inch Schmidt Sky Atlas, and go directly to the object to be observed. This will not only save telescope time but will also enable an observer to go to a new and interesting faint object without having made a photograph of that field on a previous observing run. A television system of this kind also enables an observer to stay in the data room where he can monitor the entire data-collection process instead of being at the telescope eyepiece or riding on the telescope, as is necessary with large telescopes.

\section{Photometric Devices}

For many years the photomultiplier tube has been the standard transducer for converting optical energy into electrical signals. This device has been successful because each photoelectron released by the photocathode is amplified in a noiseless amplifier with a gain of 1 million or more. The photomultiplier tube can be used in a d-c mode in which the output signal is further amplified and recorded on an analog strip-chart recorder. With the use of modern high-speed amplifiers, it has becone more advantageous to use photomultipliers in a so-called "photoncounting" mode. The variation in transit time of the electronics in a photomultiplier tube is very small, and, as a result, each photoelectron is amplified into a single electron pulse that typically has a width of 10 nanoseconds. Each electron pulse can be individually resolved and counted in an electronic counter to make the photometric measurement. Modern photomultiplier-tube photocathodes have a quantum efficiency of up to 25 percent; that is, one photoelectron is released from the photocathode for each four photons that arrive at the photocathode. This device is within a factor of 4 of being a perfect photon detector. It has been stated that the theoretical limit on quantum efficiency is 50 percent, and there is every possibility that photo- 
multiplier tubes will continue to be improved to this point. The dark count of a good photomultiplier tube is of the order of 3 counts per second, and the maximum practical counting rate is about 3 million counts per second. This means that, used in the photon-counting mode, the photomultiplier tube has a dynamic range of approximately 1 million. This mode offers further advantages in high stability and the elimination of effects due to ohmic leakage currents in the base of the photomultiplier tube.

The quantum efficiency of photomultiplier tubes is substantially higher than that of the photographic plate, but the photomultiplier tube has the disadvantage that it can only observe one point on an optical image at any one time. Because of the stability of the photon-counting techniques, it is possible to use two or more photomultipliers simultaneously to increase the observing efficiency; for example, the 200-inch telescope is equipped with a spectrophotometer which has 32 photomultiplier tubes operating simultaneously. In principle, this gives 32 times the observing efficiency as compared with a single channel-scanning spectrophotometer. Similar gains have been achieved at the Kitt Peak National Observatory with an instrument having 80 photomultipliers that operate simultaneously for measurements of the solar magnetic fields.

\section{Image Detectors}

For many years observational astronomers have dreamed of the possibility of an image detector with a large number of picture elements, each having the same photometric characteristics as a single photomultiplier tube. An instrument of this type would be limited only by the statistical noise of the photoelectrons released from a high-quantum-efficiency photocathode. Ideally, such a device should have as many picture elements as are contained on a photographic plate. There are many problems for which useful data can be obtained with $10^{5}$ to $10^{6}$ picture elements.

Modern image-intensifier tubes are a first step toward realization of this goal. These tubes have an input photocathode that converts the optical image into an electron image which is then amplified by accelerating the electrons through a high electric field. These electron images are transferred onto an electron-sensitive material or onto a phosphor screen that generates a large number of photons per electron; in this case, the final recording is done on a photographic material. The images so obtained are photoelectron-noise-limited, but have the disadvantage of requiring further analysis by a microphotometer before the data can be quantitatively reduced by a computer.

One of the first successful image detectors to operate in an all-electronic mode was developed by McNall, Robinson, and Wampler (1) of the Lick Observatory. Ford and Brown of the Carnegie Institution have also developed similar instruments (2). These devices are, however, limited to one-dimensional spectroscopic images. They consist of a combination of image-intensifier tubes and a scanning image-dissector tube. The image-dissector tube is a photomultiplier with magnetic deflection coils to scan the photomultiplier aperture over the optical image. The image dissector is presented with the output image from the intensifier tubes. One scan cycle takes slightly longer than 1 millisecond. This system divides the image into 500 independent picture elements, resulting in dwell time on each picture element of a few microseconds. The phosphor screen in the image intensifiers stores optical images for a long enough period to ensure that the image dissector will be exposed to at least one photoelectron from each exposed picture element during the image scan cycle. Initial reports indicate that these systems work very well and that the limitations imposed by the dynamic phosphor storage degrade the system effectiveness by approximately a factor of 2 ; that is, the system operates as though it were an ideal detector with one-half the usual quantum efficiency.

In principle, integrating television camera tubes coupled with image intensifiers should be capable of solving the image-detector problem. Until recently, it has not been possible to achieve adequate gain and resolution in a television camera tube that was sufficiently stable and practical for an operational instrument. The secondary electron conduction-type tube (SEC) and the image isocon have been used in detecting astronomical images with some degree of success. These systems integrate the image on the camera tube storage target. This electron image is then read into an analog recorder and digitized at a later time. Such devices show acceptable resolution and high sensitivity although they are not strictly photon-counting image detectors.

Several groups of investigators are developing image tubes that are true pulse-counting image detectors. Our project, a joint effort of the Hale and Princeton University observatories, is based on a detector that consists of an image intensifier and a silicon target vidicon tube. The gain before the storage target appears to be sufficient to give a detectable pulse on the target for each photoelectron released from the initial photocathode. As the electron reading beam sweeps over the storage target, an electron pulse is generated at each picture element that has received a photoelectron from the photocathode. A large dynamic digital memory is scanned in synchronism with the reading beam. When an electron pulse is detected at a particular picture element, the corresponding digital word in the memory is incremented by one. Each memory word consists of 16 bits or approximately 65,000 counts. This is sufficient memory capacity to record images with an accuracy of better than 1 percent photon noise. We plan to have the image-scan pattern adjustable from a thin rectangular array for spectroscopic images to a square array for direct image-recording of extended or extremely faint astronomical objects. While such a device is relatively simple in basic concept, there remain many serious technical problems to be solved before it can become operational. At this point we have no guarantee of success, but we do feel that we are tantalizingly close to realizing our dream of having an all-electronic digital image recorder.

\section{“Hard-Wired" Data Systems}

For many data display and recording systems it is entirely practical to construct a system in which the digital logic operators all have fixed relations to each other. These so-called "hardwired" data systems can be inexpensively and rapidly constructed. For large data systems, this method of implementation is seriously limited by the fact that any changes require a substantial amount of redesign and rewiring, and therefore that the system will be out of operation during the time of modification, and that the modified data system may not be able to perform the original function satisfactorily. Computer systems avoid this problem if they are designed with a future expansion capability. 


\section{Limited-Purpose and}

\section{Comprehensive Computer Systems}

With the advent of small inexpensive computers, it is now possible to construct acquisition systems for astronomical data with a simple computer and a limited number of peripheral devices. The basic processor unit can range all the way from a multichannel analyzer to a moderately sophisticated small-scale computer. They can be used for both telescope control or data acquisition. The low cost of these small computers means that they can be used as flexible system components with the same convenience as general purpose electronic counters or strip-chart recorders have been used in the past. This concept offers the additional advantage that one computer can fail without affecting other independent systems. These systems are relatively inexpensive, and can be assembled in a short period of time, but they require at least a minimum of programming skill for the operator and necessitate a direct interaction between observer and computer. Some systems have keyboards and cathode-ray tube displays to provide for the observer interface, but most require that the operator use a teletype machine for entering operational commands.

The computer systems built at the Hale Observatories are comprehensive systems that include provision for data acquisition, data reduction, and telescope control in one system. This approach has the disadvantage of higher initial cost and a lengthy construction time, but we feel that, in the loltg run, this will be more than offset by the greater flexibility and higher efficiency of operation.

At the heart of these systems is a central processing unit (CPU) and two major sets of peripheral devices. In the first set are standard devices that are attached directly to the CPU, either through the direct memory access (DMA) or the direct input-output bus (DIO). The second set are special observing devices that are attached through a special input-output interface unit.

The CPU which we are using in our current systems is a Raytheon 703 minicomputer. The word size in the 703 is 16 binary bits; this corresponds to a total number of 65,536 . The machine cycle time is 1.75 microseconds. This is classed as an intermediatespeed machine. The CPU has an instruction set of 74 commands. It has all of the nccessary controls to read any memory location or register and to enter data manually into any memory location. The CPU has provision for up to 16 levels of interrupt and power-fail protection of the memory. It also allows the operator to single step the program during "debugging."

\section{Standard Peripheral and}

\section{Special Peripheral Devices}

The systems that we have can be equipped with up to 32,000 words of fast-access core memory and up to four slow-access disk memories, each disk memory having approximately 400,000 words of storage. A teletype machine is also included as a standard peripheral device. The teletype machines have a standard keyboard and can type on a roll of paper and, in addition, punch and read from paper tape. The usual magnetic tape recorders used with these systems have nine tracks and record 800 characters per inch. Each character consists of one byte (eight binary bits) or one-half computer word plus a parity check bit. These magnetic tape units can be used for storing and loading programs as well as storing data.

The standard data output devices include a fast line printer with 132 alphanumeric characters per line. These systems can be equipped with summary punch-card readers and punches. A digital plotter can also be used to make graphic plots of the reduced data.

Hardware arithmetic units can provide any system, large or small, with a much higher speed of operation. In effect, these devices perform arithmetic operations in a few machine cycles rather than in software, which requires several hundred machine cycles. We are contemplating devices that will perform multiple precision adding, subtracting, multiplying, and dividing. Other units will convert fixed point to floating point numbers, binary to decimal numbers, and vice versa. We are also considering the incorporation of hardware square-root and trigonometric functions.

All these devices are connected to the CPU by the specialized inputoutput controller. The connection between the controller and the devices is a single serial multiconductor cable that goes from the computer to the remote parts of the telescope. Any of these devices, and any future devices, can be connected at any point along this cable. Data can be fed from any of the devices to the CPU, and the CPU can issue commands and supply data to any of the peripheral devices. Each device has provision for status or sense circuits, and also can send an interrupt signal to the CPU when data or control functions require servicing by the CPU. Our existing controller can handle over 200 independent special peripheral units.

The clock and timing circuits are incorporated in one of the most important special peripheral devices. The basic high-precision crystal oscillator operates at 5 megahertz civil time. A hardware algorithm is used to convert from a civil time base to a sidereal time base. The oscillator and conversion algorithm are accurate to about 1 part in $10^{8}$.

The two photometric data counters, the acquisition timer, and the chopper timer are located on one chassis. All of the operating functions of these circuits can be controlled by the software. The input channels accommodate photomultiplier pulse amplifiers or voltageto-frequency converters. Each counter is a binary counter with a capacity of 24 bits or approximately 16 million pulses. One counter is a standard data counter, and the other is designed to operate as a standard counter or a preset counter. This preset counter will count up to any number that has been specified by the software. The acquisition timer has a capacity of 32 binary bits or approximately $4 \times 10^{9}$ counts. The input clock to the acquisition timer is a 1-megahertz signal, thereby providing a time resolution of 1 microsecond and a maximum time of 4000 seconds. When the system is used with the preset counter as the data interval controller, the acquisition timer indicates the total lapsed time for each data-collection interval.

The chopper timer circuit has two inputs, one for pulses from magnetic pickups and the second for square waves from optical pickups. When one of the magnetic pulses occurs, or the square-wave input changes, the chopper timer opens a gate to the counter circuits. The length of time the chopper timer is opened is determined by software control. After the chopper timer has reached its preset value, it closes the data inputs; this provides a dead time between each half cycle of the chopper interval to allow for switching from one optical input to another. The phasing of the chopper cycle must be accomplished by mechanical adjust- 
ment of the pickup on the chopper wheel. The sign of the chopper signal is determined from the polarity of the magnetic pulses or the direction of change of the square-wave input. When used in the chopper mode, the data gates can open and close only at a preselected zero phase point on the chopper signal. This ensures that the data will be collected for an integer number of chopper cycles. The data counters and the acquisition timer are operated with common gate circuits, so that all three are opened and closed simultaneously.

The mechanical position of the telescope and observing instrument subassemblies are read in through one of five encoder inputs or two special circuits. The encoders measure the hour angle', declination, focus, dome, and spectrograph grating position. All of these encoders are multiturn absolute encoders. The windscreen position is determined by digitizing an analog signal, which is generated from a variable autotransformer mechanically connected to the windscreen drive. There are 32 independent auxiliary inputs that can be connected to microswitches to determine the position of filter wheels, diaphragm wheels, offset photometer bases, and other mechanical devices.

At each observing station, and at the night assistant's control desk, there is a push-button box which is used to control the entire data-collection process. There are push buttons to record identification information, start, hold (or suspend), and stop the data acquisition. There is also a switch that can be used to suppress the recording function. This box provides the observer with those, and only those, controls necessary for the data-collection process. On each button we have used a standardized three-state indicating system. If no light is turned on in the push button, the function is not available, and if the observer pushes that button, no action will occur. If the push button is illuminated with a colored light, this indicates to the observer that the function is available and can be initiated. If the button is illuminated with a white light, it indicates that the function described on the button is in operation. If the operator pushes a button that has a white light, no action will be taken by the system, since the appropriate action is already in process. This convention allows the operator to control the system and also to know the system status.
Because there are times when the illumination on the button must be greatly reduced, we have added a sounder that gives a short beep tone every time the pattern of lights on the buttons changes.

For data display we use a special computer interface unit as the source for a video signal, and this can be connected to any standard television monitor. These monitors can be placed anywhere in the dome area. The image on the television monitors contains information about the telescope operation and the data collection. This includes all of the encoder and mechanical position inputs, the clock times, the count contained in the photometric counters and the lapsed time since the data collection was started. Arbitrary information about the object can be entered on the television screen, as well as other control parameters, such as the duration of the acquisition time. The data is entered into the television image by means of a keyboard located at the night assistant's station.

The circuits, which generate the signals for the declination and right-ascension, tracking-rate motors, are contained in one chassis unit. The tracking rates can be controlled over a range of \pm 5000 seconds of arc per hour with a resolution of less than 1 second of arc per hour. All of the input frequencies come from the basic time standard and therefore are extremely precise. A switch makes the trackingrate controllers operable from either the computer or manual switches on the front panel. This feature provides for tracking-rate operation at times the computer is not available.

\section{Software System}

Although the computer was supplied with an excellent software library by the manufacturer, it has been necessary to construct an extensive assembly language program. The uniqueness of the program comes from the special requirements of astronomical observation and the fact that most of the program is concerned with special input and output devices. The program contains approximately 70 subroutines. Of these, 11 are supervisory subroutines that link and control the other subroutines. There are 35 input or output subroutines that control the data to and from the various peripheral devices, such as the television display, strip printer, clocks, counters, and timers.
Ten arithmetic subroutines are required. The remaining 14 subroutines are required for code conversion, such as binary-to-binary coded decimal, and format preparation for the various displays and output devices. Fortran language is used for the part of the program that computes the hour angle and declination-tracking rates, the refraction displacements, the dome and windscreen positions, and the air mass. We anticipate that the systems program will continue to be enlarged and revised as new observing requirements develop in the future.

\section{Solar Astronomy}

Although most of the instruments described above are related to nighttime astronomical observations, many of them are used for solar observations, although with some important differences. The light levels that are observed in solar astronomy tend to be substantially higher and, therefore, pulse-counting techniques have been less successful. Voltage-to-frequency converters and digital data counters have been used to great advantage. Solar electronic observing equipment, therefore, has more analog or directcircuit technology. Most solar observing instruments contain analog servo systems to position the solar image. The solar image is frequently scanned over the slit of a spectrograph for intensity, magnetic, or motion measurements; in other cases, circular scans around the limb of the sun are used to study the corona of the sun. Because there are many active solar events that change rapidly, there is a requirement for automatic sequencing cameras. A variety of television cameras have been used for displaying, recording, and detecting solar flares.

\section{Conclusion}

The changes resulting from this electronic revolution have an impact as great as or greater than those from any of the major instrumental developments of the past. In previous times astronomers could only hope for the observational power that can now be realized by modern electronic instrumentation.

\section{References}

1. J. McNall, L. Robinson, E. J. Wampler, Publ. Astron. Soc. Pacific 82, 837 (1970).

2. W. K. Ford, Jr., and L. Brown, Carnegie Inst. Year B. 69, 370 (1971). 